strates that an alternative structure, in which sequences for the two quinone pockets are undifferentiated, is nevertheless functional enough to support photosynthetic growth. This simplified reactioncentre structure provides an opportunity to study the directionality of electron transfer in a system in which numerous contributions to the L/M structural asymmetry have been eliminated. It offers as well a further indication that the presentday purple bacterial reaction centre could have evolved from a more symmetrical homodimeric ancestor.

\section{William J. Coleman}

Eldu Pont de Nemours \& Co,

Experimental Station, Wilmington,

Delaware 19880-0173, USA

Douglas C. Youvan

Palo Alto Institute for Molecular Medicine, 2462 Wyandotte Street, Mountain View, California 94043, USA

\section{Naming names}

SIR - Patterson ${ }^{1}$ takes exception to our usage of the terms 'bird', 'dinosaur' and 'avialian' in reference to a new Mongolian fossil archosaur, Mononykus olecranus $^{2}$. Some of Patterson's criticisms stem from misrepresentation of our paper, while others reflect fundamental disagreements. Patterson suggests that we consider Mononykus a member "of birds (a monophyletic group) and dinosaurs (a nonmonophyletic group)". We did not say this; throughout the paper we consider dinosaurs to be monophyletic because birds are members of this group. Birds are a kind of dinosaur, and Patterson's neologism "dinosaur-like birds" is therefore nonsensical.

A second issue raised by Patterson concerns the criteria for clade demarcation in groups comprising both extant and fossil taxa. Hennig ${ }^{3}$ proposed three methods for recognizing clade boundaries, the first two based on relationships and the third based on 'essential' characters. We agree with Hennig and Patterson that the third criterion leads to confusion in formal definitions of taxa. We used the term 'bird' to refer to members of the Avialae, and Patterson suggests that in so doing we used this third criterion, thus leading to confusion. Because we defined our taxonomic groups phylogenetically and pointed out that our usage of the term 'birds' was in the casual, rather than the formal taxonomic, sense, we do not see where confusion could arise.

We followed the work of Gauthier and others ${ }^{4-6}$ in considering the formal taxonomic term Aves to comprise only those taxa inferred to have descended from the most recent common ancestor of extant birds (method 1 of Hennig). Patterson instead advocates the inclusion in extant groups of all extinct taxa more closely related to the group than to its sistergroup (method 2 of Hennig). We prefer the so-called crown-group approach because, of the two methods, it most closely reflects longstanding usage of the content of Aves. The method advocated by Patterson would require that Aves include not only birds but pterosaurs and ornithischian and sauropod dinosaurs ${ }^{4.7}$. Most ornithologists would have difficulty expanding their expertise to these groups.

In advocating Hennig's method 2, Patterson suggests that method 1 may provide misleading dates for the first appearance of the group. Although a molecular biologist would be misled if she or he thought that the age of the oldest member of the crown group chronicled the split between it and its extant sister-group, this would have more to do with sloppy scholarship than with any pitfalls in method 1 . Method 2 would be similarly misleading if the molecular biologist asked a different question (for example, concerning the radiation of the living taxa rather than when they split from their sister-group). Because biologists are free to ask many different questions, we do not see that method 2 offers any advantages to guiding unsuspecting molecular biologists along the path towards enlightenment.

Finally, Patterson is concerned at the proliferation of formal taxonomic names for groups based on 'stem' species and their crown groups, such as the group Metornithes we named in our paper. We do not agree that this creates a problem, especially in an age when large databases can be manipulated with ease. Once a group is recognized to be a clade by the identification of derived characters diagnosing it, we see no justification for ignoring it merely because it includes extinct taxa. In our view, the use of a single name like Metornithes is preferable to the cumbersome "group composed of taxa $x, y$, and $z$ ". As we expand our knowledge of the biological world, names are needed to express our understanding of its diversity ${ }^{6}$. We believe that having names for all clades is more important than the practicalities Patterson prefers.

\section{Mark Norell \\ James Clark \\ Luis Chiappe}

Department of Vertebrate Paleontology,

American Museum of Natural History,

New York, New York 10024, USA

1. Patterson, C. Nature 365, 21-22 (1993).

2. Perle A., NoretI, M. A., Chiappe, L. M. \& Clark, J. M. Nature 362, 623-626 (1993); correction 363, 188 (1993)

3. Hennig, W. Insect Phylogeny (Wiley, Chichester. 1981)

4. Gauthier, J. A. Mem. Calif. Acad. Sci. 8. 1-55 (1986).

5. Gauthier, J. A., Kluge, A. G. \& Rowe, T. Cladistics 4 105-209(1988)

6. deQueiroz, K. \& Gauthier, J. A. A. Rev. ecol. Syst. 23. $449480(1992)$

Sereno, P. Mem. Soc. Vert. Pal. 2. 1-53(1991)

PATTERSON REPLIES - Norell et al. and I differ in our approach to this problem, as shown by their second paragraph above.
They object to my calling dinosaurs a nonmonophyletic group, and write "we consider dinosaurs to be monophyletic because . . . birds are a kind of dinosaur".

It is generally agreed that the main impact of cladistics is in revealing and eliminating paraphyletic assemblages ${ }^{1}$, "groups" which turn out to lack characters and reality. When dropped from scientific nomenclature, the names of paraphyletic groups are often retained merely as colloquial terms (for example, fishes, invertebrates). But there is always another possibility: a named paraphyletic assemblage can be salvaged, or made monophyletic, by expanding it to include the monophyletic group(s) whose exclusion caused the paraphyly. Reptilia (paraphyletic) might be made monophyletic by expanding it to include birds and mammals, for example, or Invertebrata might be made monophyletic by including vertebrates. One might then say "mammals (or birds) are a kind of reptile" or "vertebrates are a kind of invertebrate". Whether anything is communicated by such statements is not for me to say. But that is the procedure that Norell et al. recommend, with the difference that the paraphyletic group (dinosaurs) is extinct.

Norell et al. criticize the method I recommended $^{2}$ (the total-group) because it would force or encourage ornithologists into "expanding their expertise" to cover pterosaurs and dinosaurs, which would be included within Aves by that method. But mammalogists do not feel obliged to be expert on multituberculates, nor ichthyologists to be expert on pholidophorids, though they agree that those extinct animals are respectively mammals and teleosts. If ornithologists have to acknowledge that, when the fossil record is brought into the system, Aves includes dinosaurs and pterosaurs, I believe that the modification is less damaging than the course recommended by Norell et al., which is that ornithologists should acknowledge that their stock-in-trade is, after all, "a kind of dinosaur".

In the 150 years since Richard Owen named Dinosauria for a few bones of Iguanodon, Megalosaurus and Hylaeosaurus, the result of the efforts of palaeontologists has been to discover what dinosaurs are, not what birds are. Perhaps surprisingly, dinosaurs turn out to be kinds of bird, a theory of relationships that is most clearly and economically expressed by including them within Aves.

\section{Colin Patterson}

Department of Palaeontology,

The Natural History Museum,

London SW7 5BD, UK

1. Neison, G. in The Hierarchy of Life (eds Fernholm, B Bremer, K. \& Jörnvall, H.) 325-336 (Excerpta Medica, Amsterdam, 1989)

2. Hennig, W. Die Stammesgeschichte der Insekten (Kramer, Frankfurt, 1969)

NATURE · VOL 366 - 9 DECEMBER 1993 